\section{P2-506 INFLUENCE OF CLIMATE ON THE INCIDENCE OF URBAN LEPTOSPIROSIS}

doi:10.1136/jech.2011.142976m.33

${ }^{1} \mathrm{M}$ Carvalho, ${ }^{1} \mathrm{~S}$ Claudio, ${ }^{2} \mathrm{E}$ Boaventura, ${ }^{2} \mathrm{G}$ Ribeiro, ${ }^{1} \mathrm{M}$ Quintana, ${ }^{1} \mathrm{~A}$ Nobre, ${ }^{4} \mathrm{P}$ Diggle, ${ }^{2} \mathrm{M}$ Reis, ${ }^{2,3} \mathrm{~A}$ Ko. ${ }^{1}$ Oswaldo Cruz Foundation, Rio De Janeiro, Rio De Janeiro, Brazil; ${ }^{2}$ Oswaldo Cruz Foundation, Salvador, Bahia, Brazil; ${ }^{3}$ Yale University, New Haven, Connecticut, USA; ${ }^{4}$ Lancaster University, Lancaster, UK

Introduction Leptospirosis has emerged as an important health problem in developing countries due to the growth of slum settlements worldwide, where poor sanitation favours rat-borne transmission. Large urban epidemics occur during seasonal periods of heavy rainfall. However, a detailed analysis has not been performed to determine how rainfall, as well as other climatic factors, specifically influences the risk of leptospirosis in these endemic settings.

Methods We analysed data from 2083 leptospirosis cases which were identified during active population-based surveillance performed in the city of Salvador, Brazil between 1996 and 2010. Information on daily rainfall, humidity and temperature were obtained for the same period. A generalised additive model was fitted, using a negative binomial distribution for weekly aggregated data (729 weeks). We incorporated a non-parametric term to estimate the time trend and a sin-cosine term to control for seasonal confounding.

Results Rainfall and humidity were positively associated with the number of cases two weeks later, linearly and without a threshold. Temperature protected, even though the range between maximum and minimum temperatures is small: from 22 to $33^{\circ} \mathrm{C}$. A decreasing trend was highly significant, possibly due to intervention of sewerage and garbage collection systems.

Conclusion Leptospirosis is expected to become an increasingly important slum health problem as predicted global climate change and growth of the world's slum population evolves, and models adequate to estimate the impact of both environment and climate variables on incidence of all environmental related diseases should be incorporated in the epidemiologists toolbox.

\section{P2-507 PREVALENCE AND FACTORS ASSOCIATED TO GEOHELMINTH INFECTIONS IN CHILDREN LIVING IN MUNICIPALITIES WITH LOW HDI IN BRAZIL}

doi:10.1136/jech.2011.142976m.34

E 0 L Fonseca, M G Teixeira, E H Carmo, M L Barreto, M D C N Costa.* Federal University of Bahia, Salvador, Bahia, Brazil

Introduction The high magnitude and wide geographic distribution of parasitic infections, coupled with the negative impact that may cause the human body given to these infections have an important position among the major health problems of the population especially in developing countries. The objective of this study is to describe the occurrence of geohelminth in children of the northnortheast Brazil and to identify environmental and socioeconomic risk factors. Methods Coprologic surveys were carried out to estimate the prevalence of geohelminth infections among in children in 10 Brazilians municipalities with low Human Development Indexes. Socioeconomic and environmental data were obtained from the children's parents or guardians, and fecal samples were examined. The proportion of geohelminth infections according variables of interest was calculated. Risk factors were evaluated using multilevel logistic regression. Of the 2523 children studied, $36.5 \%$ were affected by one or more geohelminths (Ascaris lumbricoides: 25.1\%; hookworm: 15.3\%; Trichuris trichiura: $12.2 \%$ ). The overall frequency of geohelminth infections was $45.7 \%$ in rural areas and $32.2 \%$ in urban areas. Low family income $(\mathrm{OR}=1.75 ; 1.38-2.23)$, low maternal education level $(\mathrm{OR}=1.69 ; 1.39-2.06)$, presence of garbage near the home $(\mathrm{OR}=1.50 ; 1.22-1.84)$ and an increased number of individuals in the household $(\mathrm{OR}=1.41 ; 1.17-1.71)$ were associated with infection.

Conclusion The geohelminth infections were strongly related with socioeconomic conditions, stressing the importance of public interventions targeted to improve life conditions as part of its sustainable prevention.

\section{P2-508 CHANGE IN THE EPIDEMIOLOGIC PATTERN OF OCCURRENCE OF DENGUE AND DENGUE HEMORRHAGIC FEVER IN BRAZIL}

doi:10.1136/jech.2011.142976m.35

${ }^{1} \mathrm{M}$ D C N Costa, ${ }^{* 1} \mathrm{~F}$ R Barreto, ${ }^{2} \mathrm{G}$ E Coelho, ${ }^{1} \mathrm{M}$ L Ba, ${ }^{1} \mathrm{M}$ G Teixeira. ${ }^{1}$ Federal University of Bahia, Salvador, Bahia, Brazil; ${ }^{2}$ Health Ministry of Brazil, Salvador, Bahia, Brazil

Introduction In Brazil, three serotypes of dengue virus (DENV1 to 3) circulate. The incidence of dengue fever (DF) was greater in adults and was lower than the proportion of dengue haemorrhagic fever (DHF). However, since 2007 this has changed. This study describes and discusses some of the determinants of this change.

Method Data from 1990 to 2010, recorded in the Notification and Hospital Information Systems, were used. The incidence of DF and the number of hospitalisations for DHF were represented in time trends curves, by age-group.

Results Until 2000, the proportion of DHF was $0.06 \%$; In 2002 and 2009 it was $0.34 \%$ and $0.46 \%$, respectively. Between 1998 and 2006, DF and DHF were concentrated in individuals over 14 years old, particularly between 20 and 40 who represented more than $50 \%$ of the cases. In 2007, there was substantial increase in hospitalisations for DHF of individuals under 15. Between 1998 and 2006, the proportion in this age-group varied from $9.5 \%$ (1998) to $22.6 \%$ (2001, and in 2007 reached 53\% $(p<0.05)$. This trend increased in 2008, and has remained until 2010 .

Conclusions There was a significant increase of DHF and in the ratio DF/DHF in Brazil, especially by age-group pattern. A more virulent DENV-2 serotype, responsible for outbreaks from 2007 to 2008, could have occurred, but there was no change in phylogenetic DENV2 that circulates in Brazil since 1990. It is possible the sequence of the three DENV is a important factor in determining the changes in epidemiology in this country.

\section{P2-509 POPULATION IMPACT OF DEPRESSION ON FUNCTIONAL DISABILITY IN THE ELDERLY: RESULTS FROM THE “SÃO PAULO AGEING \& HEALTH STUDY" (SPAH)}

doi:10.1136/jech.2011.142976m.36

S A da Silva, ${ }^{*}$ M Scazufca, P R Menezes. University of São Paulo, São Paulo, Brazil

Introduction With the fast population ageing, disability among the elderly is becoming a major public health issue. Depression is highly prevalent in this phase of life and may be associated with a significant proportion of the disability among elderly populations. We aimed to investigate the association between depressive symptoms and functional disability in older adults, independently of comorbidities and socioeconomic factors and to estimate the Population Attributable Fraction (PAF) of disability due to depressive symptoms and depression.

Methods A cross-sectional survey was carried with 2072 residents aged 65 years or over living in a low-income area of São Paulo, Brazil. ICD-10 depression and depressive symptoms were assessed with the Geriatric Mental State and the Neuropsychiatric Inventory. The assessment of disability was made using the WHO Disability Assessment Schedule Instrument (WHO-DASII). The $90^{\text {th }}$ percentile of WHO-DAS scores was considered as high disability. 
Results The prevalence of depression and depressive symptoms were $4.8 \%$ and $21.4 \%$, respectively. Depression (OR 8.0; 95\% CI 4.2 to 14.3 ) and depressive symptoms (OR 2.7; $95 \%$ CI 1.7 to 4.2 ) were strongly associated with high disability, even after adjustment for all demographic and socioeconomic conditions and physical morbidities. Depression had a PAF of $15 \%$ (95\% CI $10 \%$ to $19 \%)$, whereas depressive symptoms had a PAF of $17 \%$ (95\% CI $9 \%$ to $24 \%$ ).

Conclusions Depression and depressive symptoms were the morbidities that contributed most to the framework of disability in the elderly. Effective management of depressive states in the elderly, delivered at the primary care level, may reduce the total population disability.

\section{P2-510 GESTATIONAL AND NEONATAL OUTCOMES IN TEENAGE PREGNANCIES}

doi:10.1136/jech.2011.142976m.37

${ }^{1} \mathrm{~A}$ Dias, ${ }^{* 1,2}$ E L Costa, ${ }^{2} \mathrm{M}$ C F Sena, ${ }^{1} \mathrm{~L}$ S Vianna. ${ }^{1}$ Botucatu Medical School, Botucatu, Sao Paulo, Brazil; ${ }^{2}$ Health Sciences School, Brasilia, Federal District, Brazil

Introduction According to the UK government, social exclusion increases the risk of teenage pregnancy and educational factors may be dimensions of such exclusion. In Brazil, around 700000 girls (32000 under 14 years) were reported to give birth in 1999.

Objective To compare gestational follow-up and neonatal outcomes in the age groups of 12-14 years (early adolescence), 15-18 years (late adolescence), and 19-35 years (adulthood), based on the number of prenatal visits, birthweight, and 5-min Apgar score.

Method Retrospective cross-sectional study conducted between 1 January 2006 and 31 December 2009, using secondary data (Livebirth statement) from the South Wing Regional Hospital, Brasília, Federal District.

Results Of 24800 newborns, the mothers of $87.06 \%$ were adults, $12.25 \%$ late adolescents and $0.69 \%$ early adolescents. The number of prenatal visits was adequate ( $\geq 7$ visits) in $85.60 \%$ of the adults, and in only $14.00 \%$ and $0.40 \%$ of the late and early adolescents, respectively, with statistically significant differences. Birthweight distribution also significantly differed among groups. In adult pregnancies, birthweight values were the highest, although mean values were within the normal range. The proportion of low birthweight was significantly higher in early adolescence pregnancies. Five-minute Apgar scores were inadequate in $4.80 \%$ of early adolescence pregnancies, $2.80 \%$ of late adolescence pregnancies, and $1.90 \%$ of adult pregnancies, with distribution also differing among groups.

Conclusion Adolescent mothers require special care during gestation as they comprise a risk group that has been neglected. The adverse perinatal outcomes observed underscore the inadequacy of gestational follow-up.

\section{P2-511 THE RELATIONSHIP BETWEEN ALCOHOL USE AND INJECTING DRUG USE: IMPACTS ON HEALTH AND SOCIAL FUNCTIONING}

doi:10.1136/jech.2011.142976m.38

${ }^{1,2} \mathrm{P}$ Dietze, ${ }^{*}{ }^{1} \mathrm{R}$ Jenkinson, ${ }^{1} \mathrm{C}$ Aitken, ${ }^{1} \mathrm{M}$ Stoove, ${ }^{2} \mathrm{D}$ Jolley. ${ }^{1}$ Burnet Institute, Melbourne, Victoria, Australia; ${ }^{2}$ Monash University, Melbourne, Victoria, Australia

Introduction Alcohol is a major risk factor for heroin overdose, but the effects of alcohol consumption on other health and social outcomes for people who inject drugs have not been systematically evaluated. In this paper we explore the effects of alcohol on health and social outcomes for people who inject drugs.

Methods Data were obtained from 655 people who inject drugs as part of the Melbourne Injecting Drug User cohort study (MIX). AUDIT-C scores $(0,1-8,8+)$ were generated and associations between these AUDIT-C scores and health and social outcomes were examined using logistic regression for dichotomous outcomes and linear regression for continuous outcomes.

Results While around 40\% of the MIX cohort reported never drinking alcohol, 43\% scored between 1 and 8 and $17 \%$ above 8 on the AUDIT-C. A score of $8+$ on the AUDIT $C$ was associated with a variety of negative health outcomes including non-fatal heroin overdose, as well as increased use of health services such as hospital emergency departments. Participants who reported drinking were more likely to report perpetrating violent crime, but were no more likely to report committing other sorts of crime such as fraud. Drinkers were more likely to report less overall life satisfaction than non-drinkers

Conclusion The rate of abstinence in the MIX cohort was high, but those who reported drinking alcohol exhibited poorer health and social outcomes. Alcohol was associated with an increase in violent offending within a population with high rates of offending more generally.

\section{P2-512 PSYCHIATRIC READMISSIONS AND THEIR ASSOCIATION WITH SOCIODEMOGRAPHIC AND CLINICAL PROFILES}

doi:10.1136/jech.2011.142976m.39

${ }^{1}$ A R F Furegato, ${ }^{*}$ 'S A Castro, ${ }^{2} \mathrm{~J}$ L F Santos. ${ }^{1}$ Escola de Enfermagem de Ribeirão Preto - USP, Ribeirão Preto, São Paulo, Brazil; ${ }^{2}$ Faculdade de Medicina de Ribeirão Preto - USP, Ribeirão Preto, São Paulo, Brazil

Introduction Despite recent advances, the frequency of psychiatric hospitalisations remains high.

Objective To identify the frequency of readmissions in a psychiatric hospital associated with patients' social demographic and clinical profiles.

Methods A structured questionnaire was applied during 2008 to collect the reports from the Psychiatric Hospital at Ribeirão Preto - Brasil

Results In 2025 records, 681 readmissions (34\%) were identified. Most of the subjects ( $99 \%$ ) came from the same region of the study, the majority were men, white, $30 \%$ aged $40-49$ and $26 \%$ up to 29 years, $23 \%$ were married. Most of the readmissions were caused only by treatment dropout (47\%) or associated with low family support (26\%). Prevalent diagnoses were schizophrenia $(27 \%)$, affective disorders (30\%) and use of alcohol and drugs (15\%). The mean age at beginning of disease was higher among men $(p=0.001)$. The length of stay in the hospital was higher for women, especially in the age group 40 to $49(p=0.032)$. Associations were found between gender and age $(p=0.007)$, marital status $(p=0.000)$, reason for hospitalisation $(p=0.002)$, physical status on admission $(p=0.004)$, hospitalisations in other psychiatric hospitals $(p=0.003)$, type of discharge $(p=0.003)$, treatment during hospitalisation and physical status at discharge $(\mathrm{p}=0.004)$.

Conclusions The flow of care is consistent with the principles of regionalisation of the Unified Health System (SUS), however, the rate of readmissions due to treatment dropout is very high, which is an epidemiological alert to all sectors involved.

\section{P2-513 SOCIAL AND GEOGRAPHICAL VARIATIONS OF MATERNAL MORTALITY IN MEXICO}

doi:10.1136/jech.2011.142976m.40

G J Gonzalez-Perez,* M G Vega-Lopez, C E Cabrera-Pivaral. University of Guadalajara, Guadalajara, Mexico

Introduction This paper analyses the trend of maternal mortality rate in Mexico according to the level of social marginalisation 